Annals of Pure and Applied Mathematics

Vol. 18, No. 1, 2018, 113-124

ISSN: 2279-087X (P), 2279-0888(online)

Published on 25 September 2018

www.researchmathsci.org

DOI: http://dx.doi.org/10.22457/apam.v18n1a16

Annals of

Pure and Applied Mathematics

\title{
Fractional Order Delayed Prey-Predator Model with Stage Structure
}

\author{
D.Sivakumar $^{1}$ and C.Loganathan ${ }^{2}$ \\ ${ }^{1}$ Department of Mathematics, Kongu Arts and Science College (Autonomous) \\ Erode - 638107, Tamilnadu, India, Mail: profsiva75@gmail.com \\ ${ }^{2}$ Department of Mathematics, Maharaja Arts and Science College \\ Coimbatore - 641407, Tamilnadu, India, Mail: clogu@rediffmail.com
}

Received 1 August 2018; accepted 20 September 2018

Abstract. This paper is concerned mainly with the study of fractional order prey-predator system with time delay analyzing stage structure system, the characteristic equation which deals with the local stability is calculated and the existence of Hopf bifurcation is derived using Routh Hurwitz strategies.

The parameter in this paper are fractional order and time delay. They deal with oscillatory behaviour of solutions and by Lyapunov global stability, dynamical value of complex system which are reviewed with incommensurate order. Atlast the numerical examples are used to validate the effectiveness of derived analytic results.

Keywords: Prey-Predator, Fractional order, Discrete delay, Bifurcation

AMS Mathematics Subject Classification (2010): 26A33, 34A08, 34K18, 37G15

\section{Introduction}

During the past three centuries, Fractional Calculus was developed by Riemann, Liouville, Grunwald and Letnikov. In the early $20^{\text {th }}$ century, this concept was most significant in scientific fields, mechanics, physics, engineering, informatics and artificial neural networks. The extensive usage of fractional calculus includes viscoelasticity, dielectric polarization, electro magnetic waves and so on.

Material and energy cannot be instantaneously transmitted to almost all the natural systems. Hence the existence of the time delay cannot be ignored. Delay Differential Equation(DDE) is a differential equation in which the derivative of the function at any time depends on the solution at previous time. The starting condition of the time delay system in which the history of the DDE is $(-\tau, 0)$. Dynamical analysis of the systems with time delays is more complex due to the non deterministic polynomial time hard (NP-hard) nature of the stability system [1]. Fractional order system describes behaviour of real physical system more truthfully than the integer order system $[4,7]$.

Cao and Xiao was derived the Hopf bifurcation analysis for a nonlinear system is a efficient approach and they investigated the periodic solution properties obtained near stationary state of systems and the issue of bifurcation for four neuron simplified bidirectional associative memory network with two time delays with the technique of Hopf bifurcation. Additionally Wei Hu et al., [9] discussed about details of Hopf 


\section{Sivakumar and C. Loganathan}

bifurcation memristor-based chaotic circuit with chaos in a fractional order delay system. They investigated the stability and bifurcation behaviors of this fractional order delayed memristor-based chaotic circuit system. Also differentiation with fractional order induces the basic and energetic computation for neuron that provides the information process.

Mathematical modeling through differential equations and simulation via computers play a significance role in the study of multi species population interactions. So many works had been already done on these predator-prey, there may be few works available on multi species interaction by considering FDE with time delay. Xu and Ma et al. [10] had been discussed the stability and Hopf bifurcation in a ratio dependent predator-prey with stage structure while the global stability and Hopf bifurcation of a predator-prey model with Holling type II functional responses. Deng et al. [1] analysed the Hopf bifurcation for a ratio dependent predator-prey system along with two different type of delays and considered the stage structure for the predator.

\section{Preliminaries and model description}

\subsection{Fractional derivative and its approximations}

Fractional calculus is the generalization of integration and differentiation to a non integer order integro-differential operator ${ }_{a} D_{t}^{\alpha}$ defined by

$$
{ }_{a} D_{t}^{\alpha}= \begin{cases}\frac{d^{\alpha}}{d t^{\alpha}} & R(\alpha)>0 \\ 1 & R(\alpha)=0 \\ \int_{\alpha}^{t}(d \tau)^{-\alpha} & R(\alpha)<0\end{cases}
$$

There are many definitions in fractional derivatives from which one of the best well known definitions of Riemann-Liouville definition is

$$
\frac{d^{\alpha} f(t)}{d t^{\alpha}}=\frac{1}{\Gamma(n-\alpha)} \frac{d^{n}}{d t^{n}} \int_{a}^{t} \frac{f(\tau)}{(t-\tau)^{\alpha-n+1}} d \tau
$$

for $\mathrm{n}-1 \leq \alpha<n$, where $\Gamma$ is the gamma function. The geometrical and physical interpretation of fractional derivative is

$$
\int_{0}^{\infty} e^{-s t}{ }_{0} D_{t}^{\alpha} f(t) d t=s^{\alpha} L\{f(t)\}-\left.\sum_{k=0}^{n-1} s^{k}{ }_{0} D_{t}^{\alpha-k-1} f(t)\right|_{t=0}
$$

for $\mathrm{n}-1 \leq \alpha<n$ where $\mathrm{s} \equiv \mathrm{i} \omega$ denote the Laplace variable

The fractional integral of order $\alpha>0$ of a function $\mathrm{y}:(0, \infty) \rightarrow R$ is given by

$$
I_{0+}^{\alpha} y(t)=\frac{1}{\Gamma \alpha} \int_{0}^{t}(t-s)^{\alpha-1} y(s) d s
$$

where the right hand side is pointwise defined on $(0, \infty)$.

$$
\begin{gathered}
{ }_{a} D_{t}^{\alpha} x_{i}(t)=-\mu_{i} x_{i}(t) \\
+\sum_{j=1}^{n} a_{i j} f_{i}\left(x_{j}(t)\right)+\sum_{k=0}^{n-1} b_{i k} g_{k}\left(x_{k}(t-\tau)\right) \quad i=1,2, \ldots, t>0
\end{gathered}
$$

$\left(H_{1}\right)$ The transfer function $f_{i}, g_{j}(j=1,2 \ldots n)$ satisfies Lipschitz condition, i.e., there exists positive constants $F_{j}, G_{j}$ such that

$$
\left|f_{i}(x)-f_{j}(y)\right| \leq F_{j}|x-y|, \quad\left|g_{i}(x)-g_{j}(y)\right| \leq G_{j}|x-y| \text {, for all } \mathrm{x}, \mathrm{y} \in R .
$$

$\left(H_{2}\right)$ It has a constants $C_{j}(j=1,2, \ldots n)$ such that the following inequalities hold: 
Fractional Order Delayed Prey-Predator Model with Stage Structure

$$
C_{j} \mu_{i}>\sum_{j=1}^{n} C_{j}\left(F_{i}\left|a_{i j}\right|+G_{i}\left|b_{i j}\right|\right) \quad i=1,2, \ldots . n
$$

Improvements of above models will provide us a new modeling method which describes the significance of fractional differential equations in the interaction of multiple species. Comparing to integer order differential equations FODEs have ability to provide precise description of the modeled mathematical problem. Predator - prey models are more significant in the modeling multi species population interactions and these interactions through integer order models have been studied by many authors $[2,5,7]$.

$\mathrm{Xu}$ et al. [10], have studied the stability with globality and entire Hopf bifurcation by considering the stage structure for predator. Also the time delay response in the predator response is considered in the system. The integer order prey-predator system is given as

$$
\begin{aligned}
& \frac{d x}{d t}=x(t)\left(r-a x(t)-\frac{a_{1} y_{2}(t)}{1+m x(t)}\right) \\
& \frac{d y_{1}}{d t}=\frac{a_{2} x(t-\tau) y_{2}(t-\tau)}{1+m x(t-\tau)}-r_{1} y_{1}(t)-D y_{1}(t) \\
& \frac{d y_{2}}{d t}=D y_{1}(t)-r_{2} y_{2}(t)
\end{aligned}
$$

In the above system $\mathrm{x}(\mathrm{t})$ represents the density of the prey with respect to time. $y_{1}(t)$ and $y_{2}(t)$ denote the densities of the immature and mature predator with respect to time, respectively. The parameter $a, a_{1}, a_{2}, \mathrm{~m}, \mathrm{r}, r_{1}, r_{2}$ and $D$ are positive constants in which $a$ is the intraspecific constant of the prey, $r$ is the instrinsic growth rate of the prey, $r_{1}$ and $r_{2}$ are the death rates of the immature and the mature predator respectively. The response function of the mature predator is denote by $\frac{a_{1} x}{1+m x}$. The capturing rate of mature represented by $a_{1} \cdot a_{2} / a_{1}$ is the rate of conversing prey into a new immature predator. The rate of immature predator becomes mature predator is denoted by non- negative parameter $\mathrm{D}$ and this rate is proportional to the density of the immature predator. The time delay due to the gestation of mature predator is consider by the constant $\tau \geq 0$. It is assumed that mature adult predators can only contribute to the reproduction of predator biomass.

In this work, we investigate a fractional order prey-predator interaction along with time delay is described by

$$
\begin{aligned}
& \frac{d^{\alpha_{1}} p_{1}}{d t}=p_{1}(t)\left(r-a p_{1}(t)-\frac{a_{1} p_{3}(t-\tau)}{1+m p_{1}(t)}\right) \\
& \frac{d^{\alpha_{2}} p_{2}}{d t}=\frac{a_{2} p_{1}(t) p_{3}(t-\tau)}{1+m p_{1}(t)}-r_{1} p_{2}(t)-\beta p_{2}(t) \\
& \frac{d^{\alpha_{3}} p_{3}}{d t}=\beta p_{2}(t)-r_{2} p_{3}(t)
\end{aligned}
$$

With the following initial conditions $p_{1}(0), p_{2}(0)>0$ and $p_{3}(t)=\varphi(t), \varphi \in[-\tau, 0], \alpha \in$ $(0,1], \varphi(t)$ is the smooth function. The parameter descriptions are same as in the integer order system.

\section{Dynamics in a fractional order predator-prey system}

3.1. Stability analysis

In this section we proceed with stability analysis of commensurate fractional order system $\left(\alpha=\alpha_{1}=\alpha_{2}=\alpha_{3}\right)$. 


\section{Sivakumar and C. Loganathan}

\subsection{Stability of the equilibrium points}

In this section, we analyse the local stability of each of feasible equilibria of the system (1) and the existence of Hopf bifurcation at the coexistence equilibrium. Equating the derivatives to the zeros and solving the system (1), one can get three distinct types of equilibria.

i. $\quad E_{0}(0,0,0)$ represents the trivial equilibrium of the system.

ii. $\quad E_{1}\left(\frac{r}{a}, 0,0\right)$ represents the predator extinction equilibrium.

iii. Further if the condition $\left(H_{1}\right) a_{2} r \beta-r_{2}(a+m r)\left(\beta+r_{1}\right)>0$ holds then the system (1) has a unique coexistence equilibrium $E_{1}\left(\overline{p_{1}}, \overline{p_{2}}, \overline{p_{3}}\right)$ where

$$
\begin{aligned}
& \overline{p_{1}}=\frac{r_{2}\left(\beta+r_{1}\right)}{a_{2} \beta-m r_{2}\left(\beta+r_{1}\right)} \\
& \overline{p_{2}}=\frac{r_{2}}{\beta} \overline{p_{3}} \\
& \overline{p_{3}}=\frac{a_{2} \beta\left[a_{2} r \beta-r_{2}(a+m r)\left(\beta+r_{1}\right)\right]}{a_{1}\left[a_{2} \beta-m r_{2}\left(\beta+r_{1}\right)\right]^{2}}
\end{aligned}
$$

The local stability of system (1) can be done based upon the Jacobian matrix of the system.

The characteristic equation of the system (1) at equilibrium state is

$$
\left|\begin{array}{ccc}
\lambda^{\alpha}-\left(\left[-a p_{1}+\frac{a_{1} p_{3} p_{1} m}{\left(1+m p_{1}\right)^{2}}\right]+r-a p_{1}-\frac{a_{1} p_{3}}{\left(1+m p_{1}\right)}\right) & 0 & \frac{a_{1} p_{1} e^{-\lambda \tau}}{\left(1+m p_{1}\right)} \\
-\frac{a_{2} p_{3}}{\left(1+m p_{1}\right)^{2}} & \lambda^{\alpha}+r_{1}+\beta & \frac{a_{2} p_{1} e^{-\lambda \tau}}{\left(1+m p_{1}\right)} \\
0 & -\beta & \lambda^{\alpha}+r_{2}
\end{array}\right|=0
$$

Then the characteristic polynomial is simplified as

$$
\lambda^{3 \alpha}+A_{1} \lambda^{2 \alpha}+A_{2} \lambda^{\alpha}+\left(A_{3} \lambda^{\alpha}+A_{4}+A_{5}\right) e^{-\lambda \tau}+A_{6}=0
$$

where

$$
\begin{aligned}
& A_{1}=r_{1}+r_{2}+\beta+2 a p_{1}-r+\frac{a_{1} p_{3}}{\left(1+m p_{1}\right)^{3}} \\
& A_{2}=r_{2}\left(r_{1}+\beta\right)+\left(r_{1}+r_{2}+\beta\right)\left[2 a p_{1}-r+\frac{a_{1} p_{3}}{\left(1+m p_{1}\right)^{3}}\right] \\
& A_{3}=-\frac{a_{2} \beta p_{1}}{\left(1+m p_{1}\right)} \\
& A_{4}=\frac{a_{2} \beta p_{1} r}{\left(1+m p_{1}\right)} \\
& A_{5}=-\frac{2 a a_{2} \beta p_{1}{ }^{2}}{\left(1+m p_{1}\right)} \\
& A_{6}=r_{2}\left(r_{1}+\beta\right)\left[2 a p_{1}-r+\frac{a_{1} p_{3}}{\left(1+m p_{1}\right)^{2}}\right]
\end{aligned}
$$

Put $\lambda=i \omega$ is a root of (4) where $\omega>0$ and $\mathrm{i}=\cos \frac{\alpha \pi}{2}+i \sin \frac{\alpha \pi}{2}$ then we substitute the expression of $\lambda$ into (4) and separate the real and imaginary parts of equation (4) gives

$$
\begin{aligned}
& \mu+\phi \cos \omega \tau+\psi \sin \omega \tau=0 \\
& \eta+\psi \cos \omega \tau-\phi \sin \omega \tau=0
\end{aligned}
$$


Fractional Order Delayed Prey-Predator Model with Stage Structure

where

$$
\begin{aligned}
& \mu=\omega^{3 \alpha} \cos \frac{3 \alpha \pi}{2}+A_{1} \omega^{2 \alpha} \cos \alpha \pi+A_{2} \omega^{\alpha} \cos \frac{\alpha \pi}{2}+A_{6} \\
& \phi=A_{3} \omega^{\alpha} \cos \frac{\alpha \pi}{2}+A_{4}-A_{5} \\
& \psi=A_{3} \omega^{\alpha} \sin \frac{\alpha \pi}{2} \\
& \eta=\omega^{3 \alpha} \sin \frac{3 \alpha \pi}{2}+A_{1} \omega^{2 \alpha} \sin \alpha \pi+A_{2} \sin \frac{\alpha \pi}{2}
\end{aligned}
$$

Suppose that (5) and (6) have roots, the expression $\cos \omega \tau$ and $\sin \omega \tau$ can be obtained as follows.

$$
\begin{aligned}
& \sin \omega \tau_{1}=\frac{\eta \phi-\mu \psi}{\phi^{2}+\psi^{2}} \\
& \cos \omega \tau_{2}=\frac{-(\phi \mu+\eta \psi)}{\phi^{2}+\psi^{2}}
\end{aligned}
$$

and

Based on (5) and (6) we derive

$$
\cos ^{2} \omega \tau_{1}+\sin ^{2} \omega \tau_{1}=1
$$

$$
\begin{aligned}
& \tau_{1}=\frac{1}{\omega_{0}}\left(\sin ^{-1}\left(\frac{\eta \phi-\mu \psi}{\phi^{2}+\psi^{2}}\right)+2 n \pi\right), n=0,1,2 \ldots \\
& \tau_{2}=\frac{1}{\omega_{0}}\left(\cos ^{-1}\left(\frac{-(\phi \mu+\eta \psi}{\phi^{2}+\psi^{2}}\right)+2 n \pi\right), n=0,1,2 \ldots
\end{aligned}
$$

Define the bifurcation point

$$
\tau_{0}=\min \left(\tau_{1}{ }^{(n)}, \tau_{2}{ }^{(n)}\right), \quad n=0,1,2, \ldots
$$

where $\tau_{1}{ }^{(n)}$, and $\tau_{2}{ }^{(n)}$ are defined by (10) and (11) respectively.

From (9) we obtain the characteristic equation gives the solution $\omega>0$ then we can solve the equation (4) as follows

$$
\cos ^{2} \omega \tau_{1}+\sin ^{2} \omega \tau_{1}=\left(\frac{-(\phi \mu+\eta \psi)}{\phi^{2}+\psi^{2}}\right)^{2}+\left(\frac{\eta \phi-\mu \psi}{\phi^{2}+\psi^{2}}\right)^{2}=1
$$

Solving, then we get

$$
\phi^{2}+\psi^{2}=\mu^{2}+\eta^{2}
$$

Substituting $\phi, \mu, \eta, \psi$ in (12) then we get

$$
\omega^{6 \alpha}+Q_{1} \omega^{5 \alpha}+Q_{2} \omega^{4 \alpha}+Q_{3} \omega^{3 \alpha}+Q_{4} \omega^{2 \alpha}+Q_{5} \omega^{\alpha}+Q_{6}=0
$$

where

$$
\begin{aligned}
Q_{1} & =2 A_{1} \cos \left(\frac{\alpha \pi}{2}\right) \\
Q_{2} & =A_{1}^{2}+2 A_{2} \cos \alpha \pi \\
Q_{3} & =2\left(A_{6} \cos \left(\frac{3 \alpha \pi}{2}\right)+A_{1} A_{2} \cos \left(\frac{\alpha \pi}{2}\right)\right) \\
Q_{4} & =A_{2}^{2}+2 A_{1} A_{6} \cos \alpha \pi-A_{3}^{2} \\
Q_{5} & =2 \cos \left(\frac{\alpha \pi}{2}\right)\left(A_{2} A_{6}-A_{3}\left(A_{4}+A_{5}\right)\right)
\end{aligned}
$$


D. Sivakumar and C. Loganathan

$$
Q_{6}=A_{6}^{2}-A_{4}^{2}-A_{5}^{2}-2 A_{4} A_{5}
$$

Lemma 1. When $\tau=0$, the equilibrium point of the fractional order system (1) is asymptotically stable if $\left(\mathrm{H}_{3}\right)$ holds.

$\left(H_{3}\right) \quad P_{1}>0, P_{2}>0, P_{3}>0$

Proof: When $\tau=0$, then the characteristic equation (4) becomes

$$
\lambda^{3 \alpha}+P_{1} \lambda^{2 \alpha}+P_{2} \lambda^{\alpha}+P_{3}=0
$$

where

$$
\begin{gathered}
P_{1}=r_{1}+r_{2}+\beta+2 a p_{1}-r+\frac{a_{1} p_{3}}{\left(1+m p_{1}\right)^{3}} \\
P_{2}=r_{2}\left(r_{1}+\beta\right)+\left(r_{1}+r_{2}+\beta\right)\left[2 a p_{1}-r+\frac{a_{1} p_{3}}{\left(1+m p_{1}\right)^{3}}\right]-\frac{a_{2} \beta p_{1}}{\left(1+m p_{1}\right)} \\
P_{3}=\frac{a_{2} \beta p_{1} r}{\left(1+m p_{1}\right)}+r_{2}\left(r_{1}+\beta\right)\left[2 a p_{1}-r+\frac{a_{1} p_{3}}{\left(1+m p_{1}\right)^{2}}\right]-\frac{2 a a_{2} \beta p_{1}{ }^{2}}{\left(1+m p_{1}\right)}
\end{gathered}
$$

When $P_{1}>0, P_{2}>0$ and $P_{3}>0$ hold, then (14) has three roots with negative real parts. Hence, the zero equilibrium point of the fractional system (1) is asymptotically stable when $\tau=0$. Then the proof is complete.

\section{Remark 1.}

The condition $\left(\mathrm{H}_{3}\right)$ are only sufficient conditions, not necessarily one. They can assure the asymptotically stable of the incommensurate fractional system (1) when $\tau=0$.

We need the following additional assumption to get the transversal condition of the existence for Hopf bifurcation:

$$
\left.\left(H_{4}\right) \quad \operatorname{Re}\left(\frac{d \lambda}{d \tau}\right)\right|_{\tau=\tau_{0}} \neq 0
$$

Differentiate (4) with respect to $\lambda$ and $\tau$ then

$$
\begin{aligned}
& \text { where } \\
& \begin{aligned}
& \alpha_{1}=-A_{3} \omega^{\alpha+1} \sin \left(\frac{\alpha \pi}{2}-\omega \tau_{0}\right)+\omega \sin \omega \tau_{0}\left(A_{4}+A_{5}\right) \\
& \beta_{1}=A_{3} \omega^{\alpha+1} \cos \left(\frac{\alpha \pi}{2}-\omega \tau_{0}\right)+\omega \cos \omega \tau_{0}\left(A_{4}+A_{5}\right) \\
& \begin{aligned}
\alpha_{2}=3 \alpha \omega^{3 \alpha-1} \cos \left(\frac{3 \alpha-1}{2}\right) \pi \\
+
\end{aligned} \\
&+2 A_{1} \alpha \omega^{2 \alpha-1} \cos \left(\frac{2 \alpha-1}{2}\right) \pi+\alpha \omega^{\alpha-1}\left(A_{2} \cos \left(\frac{\alpha-1}{2}\right) \pi\right. \\
&\left.+A_{3} \cos \left(\frac{(\alpha-1) \pi}{2}-\omega \tau_{0}\right)\right)+A_{3} \tau_{0} \omega^{\alpha} \cos \left(\frac{\alpha \pi}{2}-\omega \tau_{0}\right)+\tau_{0}\left(A_{4}\right. \\
&\left.+A_{5}\right) \cos \omega \tau_{0} \\
& \beta_{2}=3 \alpha \omega^{3 \alpha-1} \sin \left(\frac{3 \alpha-1}{2}\right) \pi+2 A_{1} \alpha \omega^{2 \alpha-1} \sin \left(\frac{2 \alpha-1}{2}\right) \pi+\alpha \omega^{\alpha-1}\left(A_{2} \sin \left(\frac{\alpha-1}{2}\right) \pi\right. \\
&\left.+A_{3} \sin \left(\frac{(\alpha-1) \pi}{2}-\omega \tau_{0}\right)\right)+A_{3} \tau_{0} \omega^{\alpha} \sin \left(\frac{\alpha \pi}{2}+\omega \tau_{0}\right)-\tau_{0}\left(A_{4}\right. \\
&\left.+A_{5}\right) \sin \omega \tau_{0}
\end{aligned}
\end{aligned}
$$$$
\frac{\lambda^{\prime}}{\tau^{\prime}}=\frac{\alpha_{1}+i \beta_{1}}{\alpha_{2}+i \beta_{2}}
$$ 
Fractional Order Delayed Prey-Predator Model with Stage Structure

Multiply and divide (15) by $\alpha_{2}-i \beta_{2}$ then we get

$$
\frac{\lambda^{\prime}}{\tau^{\prime}}=\frac{\alpha_{1} \alpha_{2}+\beta_{1} \beta_{2}+i\left(\alpha_{2} \beta_{1}-\alpha_{1} \beta_{2}\right)}{\alpha_{2}{ }^{2}+\beta_{2}{ }^{2}}
$$

Then

$$
\left.\operatorname{Re}\left(\frac{d \lambda}{d \tau}\right)\right|_{\tau=\tau_{0}, \omega=\omega_{0}}=\frac{\alpha_{1} \alpha_{2}+\beta_{1} \beta_{2}}{\alpha_{2}{ }^{2}+\beta_{2}{ }^{2}} \neq 0
$$

As the above mentioned concept, the following theorem can be as follows.

Theorem 1. Assume that $\left(H_{1}\right)-\left(H_{4}\right)$ are satisfied, for system (1), the following results hold.

(1) The zero equilibrium point is asymptotically stable for $\tau \in\left[0, \tau_{0}\right)$.

(2) The system (1) undergoes a Hopf bifurcation at the origin when $\tau=\tau_{0}$ i.e., system (1) has a branch of periodic solution bifurcating from the zero equilibrium point near $\tau=\tau_{0}$.

Remark 2. It needs to be underlined that we choose the sum of time delays as bifurcation to consider the bifurcation phenomenon of system (1) in the present paper. In fact, fractional order or system parameters also can affect the dynamical behaviours of fractional system (1), which will be studied in our future work.

\section{Numerical simulation}

In this section, we provide the stability and existence of Hopf bifurcation for commensurate fractional order predator prey system and Lyapunov global stability of incommensurate fractional order system. The parameter values chosen for the numerical simulations are a $=16, a_{1}=5, a_{2}=3, \beta=1, m=0.1, r_{1}=1 / 8, r_{2}=1 / 8$ and the initial conditions of the populations are $\mathrm{x}(0)=0.2, y_{1}(0)=0.2, y_{2}(0)=0.2$.

Table 1: Stability mature for $r=8$ and commensurate fractional order $\alpha=1$.

\begin{tabular}{|c|c|c|c|}
\hline Equilibrium & Eigenvalues & Nature & Index \\
\hline$E_{0}(0,0,0)$ & $8,-0.1250,-1.1250$ & Saddle & 1 \\
$E_{1}(0.8125,0,0)$ & $-8,0.6706,-1.9206$ & Saddle & 1 \\
$E_{2}(0.0471,0.182,1.456)$ & $-1.7841,-0.0928 \pm 0.7483 \mathrm{i}$ & Asymptotically Stable & 2 \\
\hline
\end{tabular}

Relation between matured predator and immatured predator when $\tau=0$ and various $\alpha$

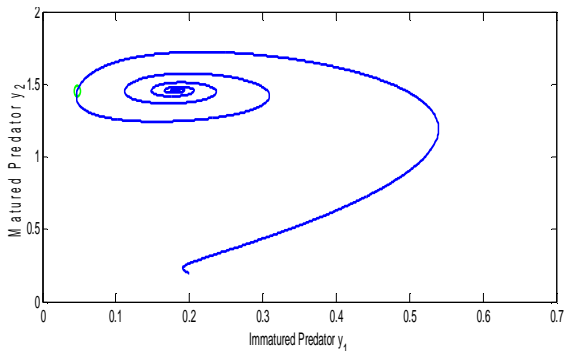

Relation between population with respect to time when $\tau=0$ and $\alpha=1$ and $r=8$

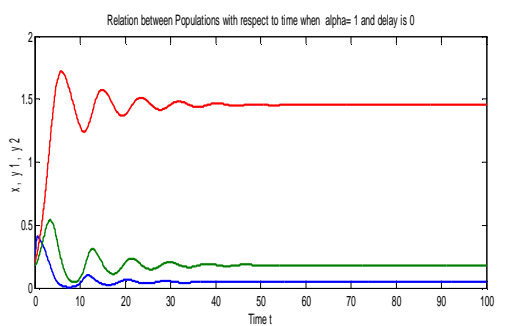

Figure 1: Stability for mature with commensurate fractional order $\alpha=1$ and $\tau=0, \mathrm{r}=8$

4.1. Commensurate fractional order $\left(\alpha=\alpha_{1}=\alpha_{2}=\alpha_{3}\right)$ and $\tau=0$

In this section, we analyse the non-delayed predator-prey system by considering the commensurate fractional order $\left(\alpha=\alpha_{1}=\alpha_{2}=\alpha_{3}\right)$ and varying the value of growth rate $r$ of prey. The equilibrium points of system (1) and the eigen values of corresponding 


\section{Sivakumar and C. Loganathan}

Jacobian matrix are given in Table 1 and Table 2. From Table 1, we can seen that $\mathrm{E}_{0}, \mathrm{E}_{1}$ are saddle points of index 1 and $E_{2}$ is asymptotically stable for $r=8$. If the growth rate of prey is increased that is $r=15$, then the corresponding equilibrium $E_{0}, E_{1}$ and $E_{2}$ and their respective eigenvalues are given in Table 2 . It can be seen that $\mathrm{E}_{0}$ and $\mathrm{E}_{1}$ are saddle points of index 1 and $\mathrm{E}_{2}$ is saddle point of index 2 .

Table 2: Stability mature for $\mathrm{r}=15$ and commensurate fractional order $\alpha=1$

\begin{tabular}{|c|c|c|c|c|}
\hline Equilibrium & \multicolumn{2}{|c|}{ Eigen values } & Nature & Index \\
\hline$E_{0}(0,0,0)$ & $15,-0.1250,-1.1250$ & Saddle & & 1 \\
$E_{1}(0.8125,0,0)$ & $-15,1.0547,-2.3047$ & Saddle & & 1 \\
$E_{2}(0.0471,0.182,1.456)$ & $-2.0047,0.0340 \pm$ \\
& $0.9968 \mathrm{i}$ & Saddle & & 2 \\
\hline
\end{tabular}

Figure 2: Unstable for mature with and commensurate fractional order $\alpha=1$ and

$$
\tau=0, r=15
$$

Hence it is clear that for the given parameter values and the fixed $r$, the system (1) will converge to fixed point for $\alpha<\bar{\alpha}$ then the system undergoes Hopf bifurcation for $\alpha=\bar{\alpha}$. Then the system shows oscillatory behaviour when $\alpha>\bar{\alpha}$ which is shown in the Figure 2. For the given set of parameter values, the stability of commensurate fractional order system can be perturbed by $\alpha$. Table 3 provides the numerical experiment on equilibrium, $E_{2}$ becomes stable or unstable by varying the fractional order $\alpha$.

\subsection{Dynamics for different $\tau$ and fixed commensurate $\alpha$}

In this section, we analyse the effect of time delay in the stability of the delayed predator prey system(1) by choosing that $\alpha$ to be fixed. In numerical simulations, the parameter and the initial values are considered as $r=8, a=16, a_{1}=5, a_{2}=3, \beta=1, m=0.1$, $r_{1}=1 / 8, r_{2}=1 / 8$ and $\mathrm{x}(0)=0.2, y_{1}(0)=0.2, y_{2}(0)=0.2$. For $\alpha=0.98$, the critical value of time delay is calculated as $\tau^{*}=0.4895$ with unique positive root $\omega_{0}=0.6325$, two pair of complex conjugates $0.2733 \pm 1.1187 \mathrm{i},-0.3444 \pm 1.0989 \mathrm{i}$ with one satisfying the condition for existence of Hopf bifurcation that is $0.2733>0$. If the value of time delay $\tau$ exceeds the critical value $\tau^{*}$ then the system undergoes Hopf bifurcation at $\tau=\tau^{*}$ which is shown in the Figure 3 and 4. Figure 5 shows that decrease in the fractional order derivative, $\alpha=0.96$ will increase the value of critical time delay $\tau^{*}=1.1$. 
Fractional Order Delayed Prey-Predator Model with Stage Structure

Table 3: Stability nature for different fractional order

\begin{tabular}{|c|c|c|c|}
\hline Parameter & Equilibrium & Eigenvalues & Nature \\
\hline$\alpha=0.97$ & $E_{2}(0.0471,0.1820,1.456)$ & $-0.0134 \pm 0.9972$ & Asymptotically Stable \\
$\alpha=0.98$ & $E_{2}(0.0471,0.1820,1.456)$ & $0.0027 \pm 0.9973$ & Unstable \\
\hline
\end{tabular}
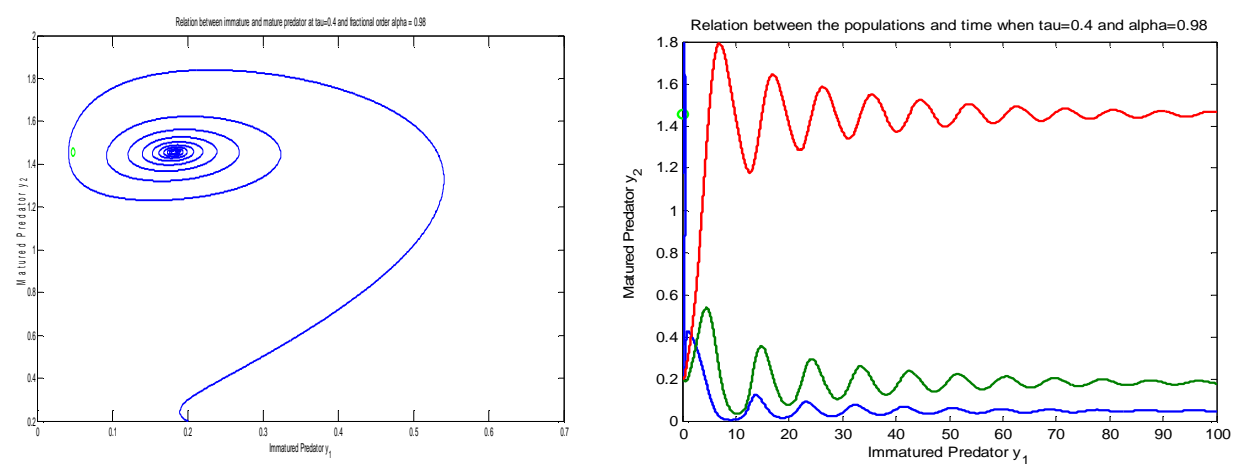

Figure 3: Stability mature for $r=8$ and commensurate fractional order $\alpha=0.98$ and $\tau=0.4$

\subsection{Incommensurate fractional order at $\tau=0$}

We will investigate the predator prey system by considering the incommensurate type of fractional order $\alpha$. The values of parameter $\mathrm{r}=15, \mathrm{a}=16, a_{1}=5, a_{2}=3, \beta=1, m=$ $0.1, r_{1}=1 / 8, r_{2}=1 / 8$ and the initial condition of the populations are $\mathrm{x}(0)=0.2$, $y_{1}(0)=0.2, y_{2}(0)=0.2$. We consider two different cases to analyse the incommensurate fractional order system (1).

\section{Case (i):}

Let $\alpha\left(\alpha_{1}, \alpha_{2}, \alpha_{3}\right)=(0.92,1,1)$ now for the given derivative orders, the equilibrium $E_{2}$ is globally as asymptotically stable.
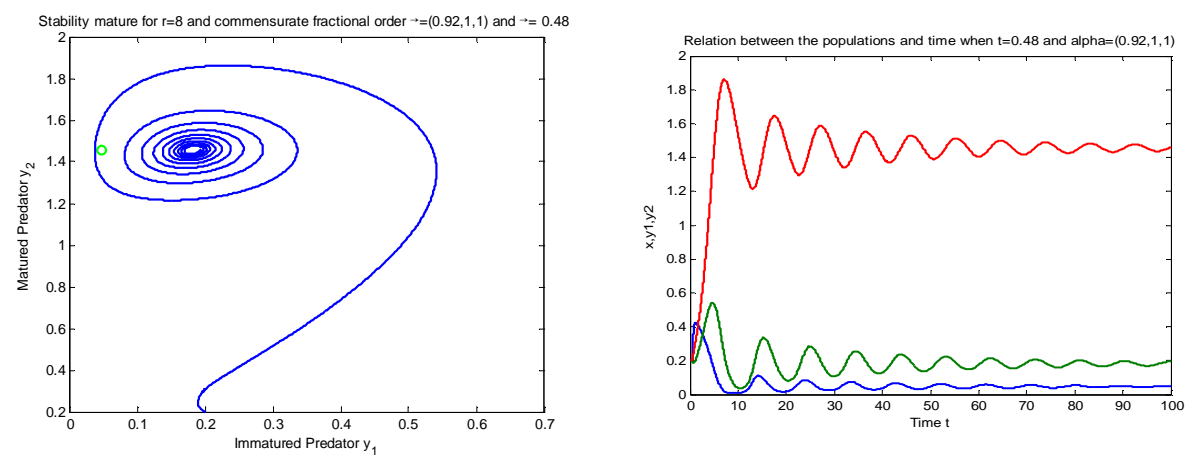

Figure 4: Stability mature for $r=8$ and incommensurate fractional order $\alpha=(0.92,1,1)$ and $\tau=0.48$ 


\section{Sivakumar and C. Loganathan}

Case (ii):

Let $\alpha\left(\alpha_{1}, \alpha_{2}, \alpha_{3}\right)=(0.94,1,1)$ by above method the system (1) does not satisfy the asymptotically stability condition. Hence the system (1) is unstable. Further the same method can be used to check for all other values of fractional orders. The pictorical representation of case( i) and case (ii) are displayed in Figure 6.
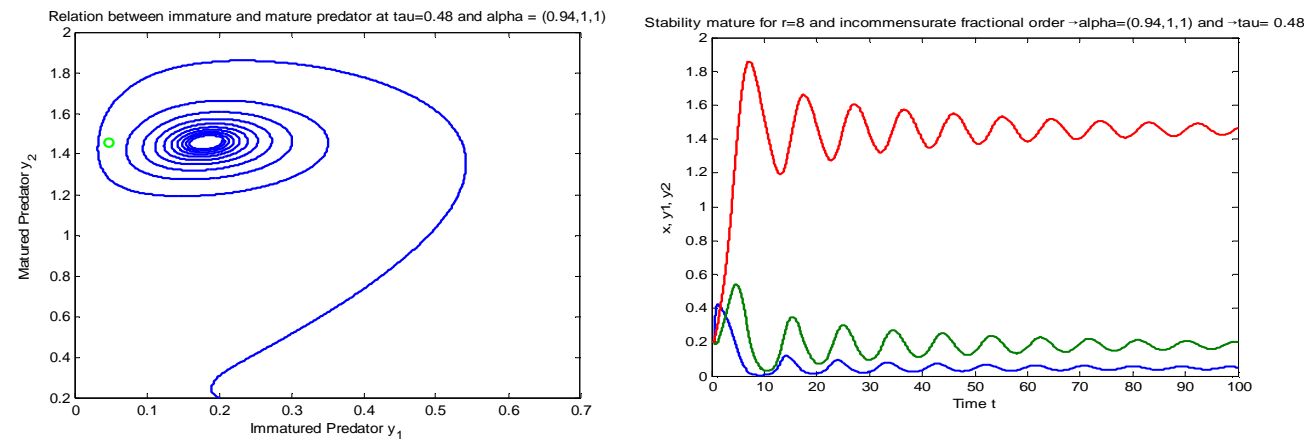

Figure 5: Unstable mature for $r=8$ and incommensurate fractional order $\alpha=(0.94,1,1)$

$$
\text { and } \tau=0.48
$$

\subsection{Dynamics for incommensurate fractional order with fixed $\tau$}

We do not provide explicit expression for a critical magnitude of $\tau$ for stability, but we provide information about the existence of such values. Figure 6 depicts the stability and periodical solution of incommensurate fractional order by considering the time delay as constant.
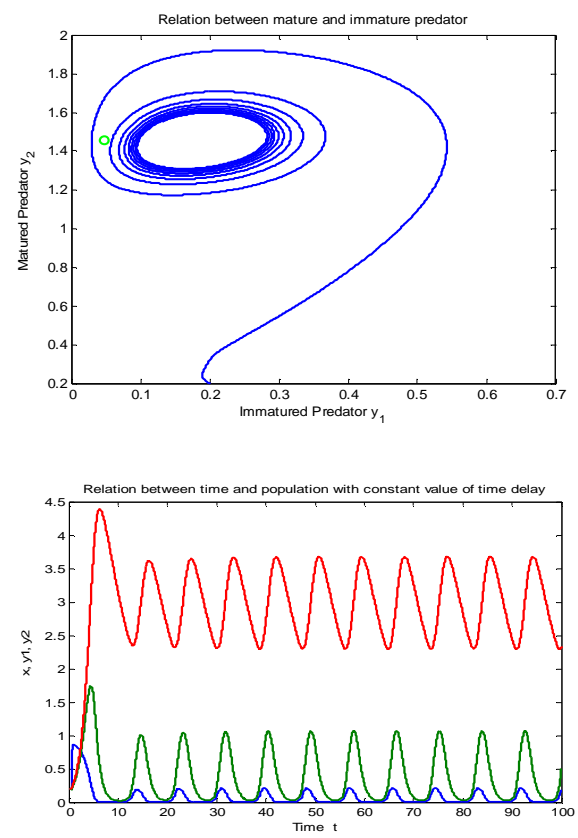

Figure 6: Solutions of the system (1) undergoes Hopf bifurcation when the value of time delay exceeds its critical value that is, $\tau=0.7>0.4882\left(\tau^{*}\right)$ and become stable for decreasing the fractional order. 


\section{Fractional Order Delayed Prey-Predator Model with Stage Structure}

\section{Conclusion}

In this work, we have developed a theoretical framework that includes sufficient biological complexity to accurately describe the dynamics of multi-species interaction. The complex dynamics of a proposed fractional order stage structured predator-prey system with and without time delay have investigated in detail via numerical simulations. We have studied the stability of trivial equilibrium and predator extinction equilibrium through the characteristic polynomial and fractional order Routh-Hurwitrz criterion. The signification of incorporating the delay into the system when it exceeds its derived critical value. Also the stability of incommensurate fractional ordered predator prey system has been discussed. The complex dynamics of both commensurate and incommensurate fractional order system have been analyzed with varying the respective fractional order as well as time delay. Also we discussed the fractional order predator prey with stage structure for the predator and time delay.

\section{REFERENCES}

1. L.Deng, X.Wang and M.Peng, Hopf bifurcation analysis for a ratio-dependent predator prey system with two delays and stage structure for the predator, Applied Mathematics and Computation, 231(0) (2014) 214-230.

2. H.Hu and L.Huang, Stability and Hopf bifurcation in a delayed predator-prey system with stage structure for prey. Nonlinear Analysis: Real World Applications, 11(4) (2010) 2757-2769.

3. C.M.Ionescu, J.A.T.Machado and R.De Keyser, Modeling of the lung impedance using a fractional-order ladder network with constant phase elements, IEEE Transactions on Biomedical Circuits and Systems, 5(1) (2011) 83-89.

4. C.Loganathan, D.Sivakumar and M.Prakash, Dynamical analysis of a fractional order delayed prey-predator model with stage structure, International Journal of Mathematics and its Applications (2018).

5. Z.H.Ma, Z.Z.Li, S.F.Wang, T.Li and F.P.Zhang, Permanence of a predator-prey system with stage structure and time delay, Applied Mathematics and Computation, 201(1) (2008) 65-71.

6. C.Pinto and J.A. Tenreiro Machado, Fractional model for malaria transmission under control strategies. Computers \& Mathematics with Applications, 66(5) (2013) 908916.

7. X.X.Qiu and H.B.Xiao, Qualitative analysis of Holling type II predator-prey systems with prey refuges and predator restricts, Nonlinear Analysis: Real World Applications, 14(4) (2013) 1896-1906.

8. O.Toker and H.Ozbay, Complexity issues in robust stability of linear delaydifferential systems, Mathematics of Control, Signals and Systems, 9(4) (1996) 386400.

9. Wei Hu, Dawei Ding, Yaqin Zhang, Nian Wang and Dong Liang, Hopf bifurcation and chaos in a fractional order delayed memristor-based chaotic circuit system, School of Electronics and Information Engineering, Anhui University, Hefei 230601, China.

10. R.Xu, and Z.Ma, Stability and Hopf bifurcation in a ratio-dependent predator prey system with stage structure, Chaos, Solitons \& Fractals, 38(3) (2008) 669-684. 
D. Sivakumar and C. Loganathan

11. R.Xu, Global dynamics of a predator prey model with time delay and stage structure for the prey, Nonlinear Analysis: Real World Applications, 12(4) (2011) 2151-2162. 